\title{
A introdução das artes nos currículos médicos ${ }^{*}$
}

Ricardo Tapajós 1
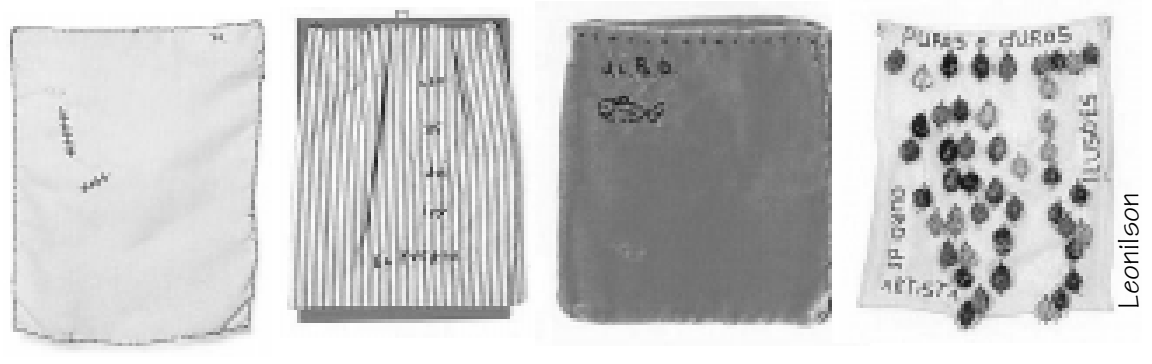

TAPAJÓS, R. Introducing the arts into medical curricula, Interface - Comunic, Saúde, Educ, v.6, n.10, p.27-36, 2002.

Appropriate professional training in the field of infection by HIV (Aids) assumes that treatment and care will be provided in a suitable, ethical and humane manner. Concern with such training has heightened the discussion on how medical training can integrate the acquisition of technical excellence with humanistic traits. Thus, Medical Education starts to incorporate the challenge of adapting itself in such a way as to provide physicians (the end product of its curricula) with both a humanistic and humane education. The Humanities, and specifically the Arts, are disciplines that classically accomplish this function. We postulate and argue that they should be introduced into medical curricula, whether due to their intrinsic value, as a source of aesthetic experience and knowledge, or whether because they can facilitate the achievement of broader objectives within these curricula.

KEY WORDS: Medical education; humanism; art; HIV/AIDS.

A capacitação profissional apropriada no campo da infecção pelo HIV/Aids prevê que tratamento e cuidados sejam oferecidos de maneira adequada, ética e humanizada. A preocupação com esta capacitação tem acentuado a discussão sobre como a formação médica pode integrar a aquisição de excelência técnica e traços humanistas. Assim, a Educação Médica passa a incorporar o desafio de adequar-se, para providenciar aos médicos, produtos finais de seus currículos, uma formação humanista e humanizadora. As Humanidades, em específico as Artes, são disciplinas que classicamente cumprem esta função. Postula-se e discute-se que elas sejam introduzidas nos currículos médicos, seja pelo seu valor intrínseco, fonte de experiência estética e conhecimento, seja para facilitar a execução de objetivos mais amplos dentro desses currículos.

PALAVRAS-CHAVE: Educação médica; humanismo; arte; HIV; Síndrome de imunodeficiência adquirida.

\footnotetext{
* Texto produzido a partir de: TAPAJÓS, R. Teaching and learning in medical Humanities: design and elective course on HIV/ AIDS in the Visual Arts. Chicago, 2000. Dissertação (Mestrado) University of Illinois.

${ }_{1}$ Médico, Supervisor da Divisão de Clínica de Moléstias Infecciosas e Parasitárias do Hospital das Clínicas da Faculdade de Medicina da Universidade de São Paulo/USP. <ritapajos@zipmail.com.br>
} 


\section{Introdução}

Educadores médicos têm estado preocupados com a capacitação profissional apropriada e efetiva no campo da infecção pelo HIV/Aids, de forma que tratamento e cuidados possam ser oferecidos de maneira adequada, ética $e$ humanizada. Esta preocupação advém do fato de que alguns estudantes de Medicina, médicos e outros profissionais da saúde têm-se mostrado relutantes em cuidar desses pacientes (Marshall e O'Keefe, 1995). A necessidade de implementação, em escolas médicas, de uma educação ampla $e$ inclusiva sobre Aids é proeminente. Entretanto, os esforços de ensino em currículos médicos tradicionais parecem devotar-se unicamente à Biologia $e$ à Epidemiologia da infecção. Cursos curriculares que carreguem a potencialidade de evidenciar, abordar e corrigir atitudes negativas $e$ preconceituosas em relação ao paciente com HIV/Aids se fazem necessários (Marshall e O'Keefe, 1995).

Com essas preocupações, a partir do ano letivo de 2000, o Departamento de Moléstias Infecciosas e Parasitárias (DMIP) da Faculdade de Medicina da Universidade de São Paulo, campus São Paulo (FMUSP), tem oferecido regularmente dois cursos inusitados a seus alunos. São propostas que pretendem ensinar aspectos da Medicina por meio do ensino de Artes. Originalmente com dois créditos cada um (um crédito é igual a quinze horas-aula), os cursos MIP-518 e MIP-519 são integrantes do elenco de cursos opcionais e podem ser cursados por alunos que os escolham a partir do segundo ano curricular. Ambos tratam das representações da infecção pelo HIV/Aids nas Artes. O primeiro abrange as representações nas Artes Visuais (Artes Plásticas, Gráficas e Fotografia), enquanto o segundo focaliza exclusivamente as Artes Cinemáticas. Dado o sucesso de sua implementação, as disciplinas foram ampliadas em carga horária e conteúdo, passando a ser oferecidas, a partir de 2002, com os códigos MIP-526 (três créditos), abordando as Artes Cinemáticas e MIP-525 (cinco créditos), ampliada para abordar as representações da infecção pelo HIV/Aids não só nas Artes Visuais, como também nas Literárias (Prosa, Poesia e Drama).

Há incontáveis doenças e condições patológicas representadas nas Artes. Tuberculose, transtornos psiquiátricos, aborto, peste, sífilis, condições dermatológicas, mal de Hansen, neoplasias, trauma e doenças degenerativas são alguns exemplos de representações bastante prevalentes, que se qualificariam como tema e conteúdo de cursos médicos centrados nas Artes. As representações da infecção pelo HIV/Aids nas Artes não são exceção. Ao contrário, a intersecção entre Aids e Artes é bastante rica. Há muitos artistas cujas vidas foram sensivelmente tocadas de alguma maneira pela epidemia. Podem-se citar David Wojnarowicz, Keith Haring, Derek Jarman, Masami Teraoka, Frank Moore, Leonilson (Artes Plásticas), Robert Mapplethorpe, Nicholas Nixon, Rosalind Solomon, Nan Goldin (Fotografia), John Greyson e Cyril Collard (Cinema), entre outros tantos, e Paul Monette, Hervé Guibert, Larry Kramer, Tony Kushner, Sarah Schulman, Susan Sontag e Edmund White, também entre muitos outros, nas Artes Narrativas. Há, ainda, inumeráveis trabalhos artísticos que abordam a doença e seu impacto na humanidade, nos mais variados meios e técnicas, incluindo as pertinentes às 
Artes Visuais, Cinemáticas e Literárias. Reproduções desses trabalhos de arte são comercialmente disponíveis em formato de vídeo, em livros de Arte e livros de literatura, portanto acessíveis a professores e alunos.

Sobre essas obras, sua iconografia, seu simbolismo, seu contexto histórico-social e seus autores há um corpo acadêmico de conhecimento $e$ crítica em constante crescimento. Este corpo de conhecimento é, per si, relevante a alunos de Medicina e torna-se ainda mais relevante para eles na medida em que toca também em outros campos de conhecimento que têm seus próprios arcabouços intelectuais e teóricos, como História da Arte, Crítica da Arte, Estética, História da Medicina, Sociologia, Antropologia e Crítica Literária.

Nessas obras de Arte, as representações da infecção pelo HIV/Aids são extremamente pertinentes às questões quotidianas do médico. Em ambas prevalecem pacientes, familiares e médicos, assim como procedimentos médicos, profissionais de saúde e ambientes hospitalares. O estudante de Medicina pode ter, assim, acesso a vários modelos (positivos e negativos) de relação médico-paciente $e$ a valiosas narrativas em primeira pessoa de experiências com a doença e percepções sobre a mesma, seu manejo e suas repercussões.

Mais do que impregnadas de quotidiano médico, essas representações são permeadas por aspectos cruciais do fenômeno humano, como morte, destino, sofrimento, transcendência, ritos, luto, preconceito, religiosidade, sexualidade, respostas sociais, políticas de Saúde, entre tantas outras. A infecção pelo HIV/Aids evoca tantas respostas em termos de comportamento humano que sua história, sozinha, tem elencado os mais variados aspectos da natureza humana. É este conhecimento da natureza humana que as disciplinas propostas procuram instilar nos alunos.

Este tipo de conhecimento, produzido pelas Humanidades, é singular e não científico em essência. É diverso daqueles conhecimentos científicos com que educadores e alunos estão acostumados a trabalhar nos currículos médicos. Sendo diverso, requer abordagens curriculares diferenciadas.

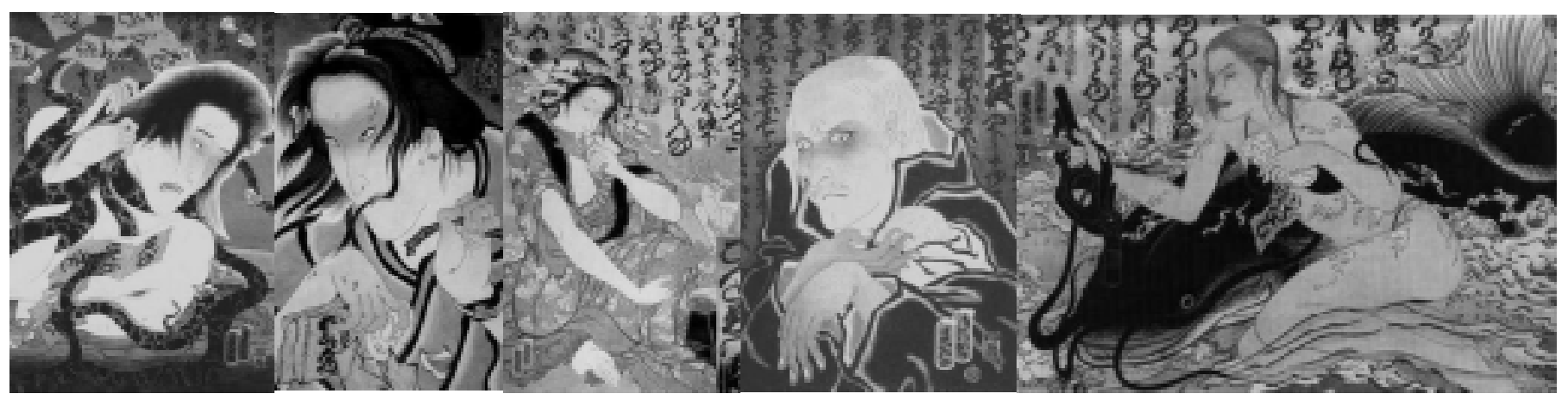

TERAOKA, Série Aids 


\section{As Humanidades nos currículos médicos}

No geral, as Humanidades são disciplinas que assistem os alunos na articulação de sentimentos e crenças, no seu processo de autopercepção, na percepção da alteridade do mundo, na percepção de diferentes comportamentos e potencialidades humanas e no entendimento dos valores que fazem de pessoas juntas uma comunidade. As Humanidades instilam sabedoria. Lidam com o que significa ser humano e viver uma vida humana. Apontam para o que as pessoas experimentam, para como elas interpretam o mundo e respondem a ele, para o sentido que elas fazem dele e para qual seja a experiência universal de humanidade e raça humana (Wilson, 1974; Hardison, 1977; Bruderle e Valiga, 1994; Darbyshire, 1994).

As Humanidades Médicas são disciplinas aplicadas a questões gerais ou particulares da práxis médica (Portugal-Alvarez, 1991). Entre elas figuram todas as Artes, a História Médica, Religião, Língua e Comunicação e Filosofia Médica (com os campos da Ética, Bioética, Epistemologia e Estética) (Portugal-Alvarez, 1991; Bruderle, 1994; Darbyshire, 1994; Taylor, 1995; Hook, 1996; Malacrida et al., 1998).

A Medicina ocupa-se de aplicar conhecimentos, inclusive científicos, a pacientes e grupos populacionais específicos, que, por serem fenômenos humanos, não são passíveis de incondicional generalização. A práxis médica incorpora, portanto, a visão científica (a biologia do estar vivo e da máquina humana Cartesiana) e visões mais abrangentes que reconheçam construtos biopsicossociais e estéticos. A Educação Médica passa, então, a ter de se preocupar em formar médicos detentores não somente de técnica impecável, como também cuidadores humanizados, sensíveis, preparados para lidar consigo e com seus pacientes, tarefa que exige trabalhar com os mais diversos valores inseridos em complexos contextos históricos, culturais e sociais. A inserção das Humanidades em currículos de Profissionais de Saúde responde a esta necessidade de humanização do produto final desses currículos. Esta inserção tem sido uma preocupação constante na literatura mundial (e, particularmente, na norte-americana) nos últimos quarenta anos, abrangendo experiências em Terapia Ocupacional, Odontologia, Enfermagem e Medicina (nesta última em áreas tão diversas quanto Medicina de Família, Cirurgia Plástica $e$

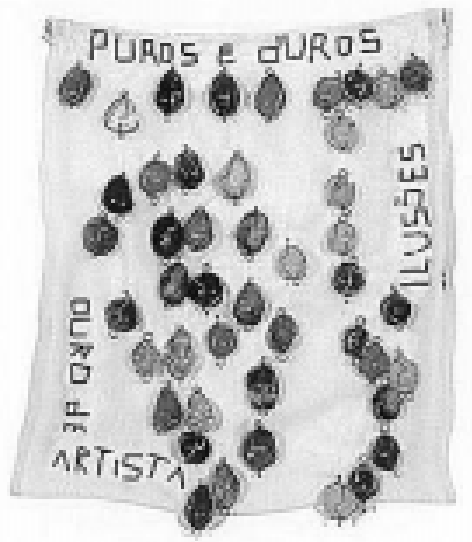

LEONILSON, Puros e Duros, 1991, Coleção Família Bezerra Dias, SP Neurocirurgia). No Brasil, esta preocupação apenas começa a aparecer de maneira sistemática.

No caso específico das Artes, a literatura de Educação em Profissões de Saúde é também prolífica em comunicar tentativas de inserção de experiências artísticas nos seus currículos. Muitos cursos são descritos, alguns dedicados às Artes Visuais (Bergman et al., 1982; Biggerstaff et al., 1984; Hoshiko, 1985; Bresnahan \& Hunter, 1989; Hogg, 1989; Loden, 1989; Davis, 1992; Darbyshire, 1994), outros, às Artes Cinemáticas (Bresnahan \& Hunter, 1989; Dichi, 1994; Crellin \& Briones, 1995). Há, ainda, cursos multi-arte que 
incluem as Artes Visuais e Cinemáticas (Moore, 1976; 1977; Bertman \& Marks, 1985; Middleton et al., 1993; Peden E Staten, 1994; Vande-Zande, 1995).

Na maioria dos casos, o estudante é observador da Arte. Em alguns casos, é também chamado a produzir obras. As Artes Narrativas, como Drama, Prosa e Poesia, tendem a predominar, mas o papel das Artes Visuais $e$ Cinemáticas é também relevante, como se verá a seguir.

\section{As Artes nos currículos médicos}

Tem havido um interesse crescente na interação entre a Medicina e as Artes, que se reflete na existência de considerável literatura sobre o assunto e na descrição de várias tentativas de inserção de poemas, peças, contos, novelas e pinturas como base para o ensino de alunos de Medicina (Sweeney, 1998). Estas muitas interações entre disciplinas da Medicina e das Artes derivam de pelo menos quatro aspectos fundamentais, quais sejam: o médico enquanto artista, o paciente enquanto artista, as questões de interesse médico direto $e$, por fim, o "entendimento mais profundo" das motivações, atitudes $e$ emoções humanas que um conhecimento das e nas Artes pode produzir (Downie, 1998; Evans, 1998; Sweeney, 1998).

Os dois primeiros aspectos podem ser tratados conjuntamente, já que tanto médicos quanto pacientes têm coisas a dizer sobre assuntos pertinentes à Medicina e podem preferir expressar-se pelas Artes. Muitos autores mostram claras influências de suas patologias reais na sua obra, tanto nas Artes Plásticas (Frida Kahlo e suas seqüelas corporais, Paul Klee e sua condição dermatológica, Keith Haring e Aids), como no Cinema (Derek Jarman e Cyril Collard, em termos de Aids) e na Fotografia (Robert Mapplethorpe e Aids).

O terceiro aspecto compreende áreas de interesse médico direto, como representações artísticas de doenças e experiências de vida relacionadas às doenças, como sofrimento, exclusão social e morte. $\mathrm{O}$ artista plástico Masami Teraoka dedica parte de sua obra à representação da Aids e sua profilaxia. O fotógrafo Nicholas Nixon retrata o morrer de Aids mesmo em vida, enquanto os retratos de Carolyn Jones mostram pessoas vivendo com o vírus. Por milhares de anos, os seres humanos têm pintado, desenhado e esculpido seus medos mais obscuros, na tentativa de controlar ou atenuar perigos (Pechansky et al., 1989). Expor estudantes de Medicina às Artes significa expô-los a uma gama enorme de medos, sentimentos e emoções que alguma vez habitaram o mundo de um artista (Noll et al., 1989; Pechansky et al., 1989) ou a vida interna de um paciente (Hardison, 1977). Os alunos podem, ainda, partilhar dessas experiências de uma maneira bastante protegida e não ameaçadora (Bruderle, 1994). Por representarem a condição humana, as Artes Visuais expõem os alunos a uma variedade de experiências humanas que são de relevância central para a prática médica, entre as quais citam-se doença, limitação, vulnerabilidade, tristeza, luto, a natureza da sociedade humana, a capacidade curativa de rituais $e$ lembranças, morte e corporalidade.

O quarto aspecto mencionado diz respeito a um "entendimento mais 
profundo". Formas distintas de conhecimento existem. Conhecimentos no campo empírico ou científico são generalizáveis e reprodutíveis, enquanto conhecimentos no campo estético são singulares (Phenix, 1964). A Medicina baseia-se em pelo menos dois tipos de conhecimento bastante distintos entre si, quais sejam: um conhecimento "conceitual" que pode ser passado de pessoa a pessoa por meio das palavras, e um "conhecimento não conceitual ou sensual" (conhecimento experiencial ou estético) que só pode ser adquirido pela interação física com o objeto a ser conhecido (Arnold et al., 1984). O conhecimento experiencial é aquele entendimento que não é possuído por aqueles cujas experiências tenham sido outras (Beckingham, 1982). Experiência em uma situação traz expertise (Lafferty, 1997). Uma pessoa descobre o que pensa sobre um dilema parcialmente por perceber aquilo que sente. Esta resposta emocional não é um meio para o conhecimento experiencial, mas constitui parte do próprio conhecimento (Hawkins, 1994). Quando quer que tome decisões, morais ou não, um clínico deve envolver todas as faculdades mentais, ou seja, razão, intuição, emoção e imaginação (Hawkins, 1994). No ensino médico, à medida que os alunos caminham da Filosofia para a Religião, para a Literatura, a Poesia, o Drama, as Artes Plásticas e a Música, tornam-se mais sensíveis à sensualidade e sensorialidade (Arnold et al., 1984), mais susceptíveis ao conhecimento experiencial ou estético.

As experiências de cunho estético dão-se de maneira epifânica, como pequenas revelações no quotidiano (Hawkins, 1994), mas também são facilitadas por situações vividas vicariantemente pelos alunos e/ou por respostas empáticas que desenvolvem. No primeiro caso, sabe-se que a experiência de ter uma doença é estranha à maioria dos estudantes de Medicina. As Artes são veículos que equipam os alunos com familiaridade a respeito desta e de outras questões não menos dolorosas, sem que eles tenham de vivenciá-las diretamente (Wilson, 1974; Bruderle, 1994). Por meio das Artes, profissionais de saúde podem alcançar, de maneira vicária, um entendimento sobre as condições humanas que não poderiam vivenciar de outra maneira (Pellegrino, 1984; Bartol, 1986; Mohr, 1995; Sweeney, 1998). No segundo caso, as Artes são úteis em colocar problemas particulares numa perspectiva que encoraja respostas empáticas (Blizek $\varepsilon_{r}$ Finkler, 1977). Esta assunção repousa em três aspectos comuns tanto às Artes quanto à noção de empatia, ou seja, resposta, emoção e conexão. A Arte evoca uma resposta que é profunda e pessoal, e esta resposta é freqüentemente emocional e conduz a conexões significativas (Peloquin, 1996). Em ambos os casos, portanto, as Artes favorecem ao aluno a aquisição de conhecimentos singulares.

Este "entendimento mais profundo" gerado pelas Artes não é mediado exclusivamente por experiências puramente estéticas, mas também por habilidades interpretativas. Teóricos na interface da Medicina e das Artes têm sugerido que a interpretação, como atividade literária, tem analogia com a prática clínica da Medicina (Gogel \& Terry, 1987) - o médico agindo como o leitor e o paciente como o texto a ser interpretado. O paciente é portador de narrativas verbais, mas também carrega formas não verbais de narrativa, como expressões, posturas, temperamentos, gestos e achados 
físicos. Assim, além de texto a ser interpretado, o paciente é também quadro, pintura, fotografia, escultura a ser apreendido. Interpretar significa construir, a partir de um sistema de signos (texto/obra), alguma coisa maior do que a presença física deste

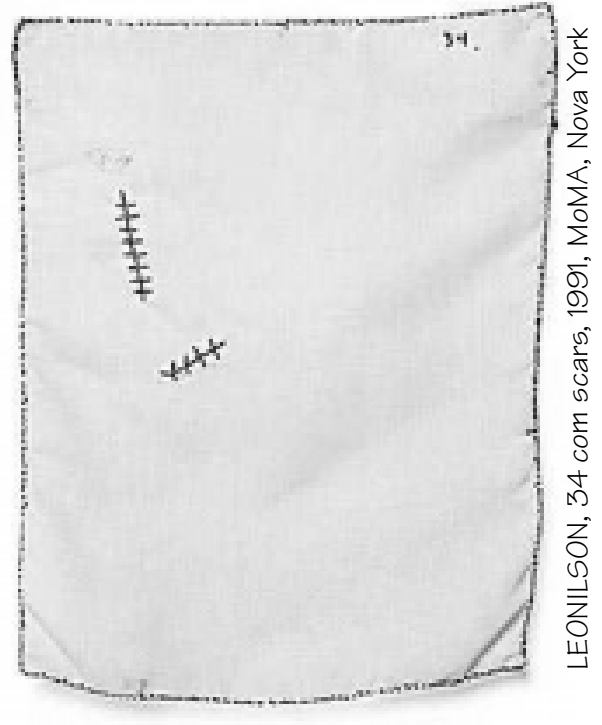
mesmo sistema. Avaliar pacientes

requer habilidades que o bom interpretador possui, como a capacidade de integrar fenômenos isolados (sejam achados físicos do paciente, sejam símbolos e metáforas no texto/obra), para produzir um sentido maior e organizá-lo numa narrativa conclusiva que permita o entendimento de uma história no contexto de outras histórias do mesmo narrador (Taylor, 1995). Construir significado clínico a partir de narrativas e achados físicos é tarefa interpretativa (Gogel \& Terry, 1987), chamada pela Medicina de diagnóstico (Brieger, 1994), ou seja, a "leitura" do texto/obra que o paciente e sua doença perfazem juntos (Evans, 1998). Se a Medicina pode ser assim concebida como um campo interpretativo ou hermenêutico (Gogel \& Terry, 1987), as Artes e a disciplina correlata de Crítica de Arte podem oferecer contribuições valiosas aos estudantes de Medicina.

A inclusão das Artes Visuais nos currículos médicos parece, portanto, desejável (Downie, 1998). Elas incentivam e oferecem oportunidades para reflexão crítica, pensamento não-científico, pensamento simbólico, reações estéticas, liberação de sentimentos, consciência de valores pessoais $e$ sentimentos, observação, análise e outras habilidades hermenêuticas. Acresça-se que elas incentivam habilidades intelectuais e psicomotoras específicas úteis para a profissão, como visão espacial $e$ a capacidade de pensar em terceira dimensão. Por fim, as Artes Visuais são recreativas, na medida em que produzem prazer e um contra-ponto relaxante ao stress diário (Siegel \& Siegel, 1977; Parkin, 1987; Morani, 1992; Pásztor, 1993; Vogel, 1993; Bruderle \& Valiga, 1994; Darbyshire, 1994; Taylor, 1995; Hook, 1996; Bertman, 1998; Malacrida et al., 1998).

Discutidas algumas razões que justificam a presença das Artes nos currículos médicos, cabe ressaltar que educadores de Arte advogam a presença das Artes em currículos gerais por meio de duas classes não mutuamente exclusivas de argumentos (Dobbs, 1998). A perspectiva Essencialista prega a noção de que as Artes devem existir nos currículos pelo seu valor intrínseco e por aquilo que elas provêem, ou seja, uma fonte de experiências estéticas, de conhecimento e entendimento de si mesmo, dos trabalhos de Arte e do mundo onde eles foram criados (Dobbs, 1998), além 
de desenvolvimento de cultura estética e apreensão de metáforas visuais (Smith, 1989). A perspectiva Instrumentalista valoriza a Arte pela sua contribuição para o cumprimento de objetivos mais gerais em outras áreas $e$ disciplinas não artísticas. Exemplos seriam o desenvolvimento de percepção de uma sociedade multicultural, competência em comunicação, desenvolvimento cognitivo, imaginação, julgamento, capacidade de resolução de problemas e pensamentos de complexidade intelectual superior (Dobbs, 1998). A Educação pelas Artes carrega, então, um valor intrínseco $e$ um valor extrínseco utilitário, ambos contribuindo para uma educação generalista e liberal.

A finalidade com a qual as Artes são introduzidas nos currículos médicos pode obedecer potencialmente a uma, a outra ou a ambas as perspectivas (Essencialista e Instrumentalista). Atendo-se à perspectiva Instrumentalista, educadores médicos podem argumentar que o ensino/aprendizagem das $e$ nas Artes em currículos médicos carrega a função de facilitar a execução de outros objetivos mais amplos dentro desses currículos, contribuindo para a formação de um médico detentor não só de excelência técnica, mas também dos traços humanistas instilados por uma Educação Geral e requeridos pelo paradigma Humanista da Medicina.

Poder-se-ia, então, dizer que o ensino/aprendizagem das Artes pode mediar o ensino/aprendizagem da própria Medicina, ou seja, pode-se ensinar Medicina por meio das Artes. É esta hipótese que alimenta e sustenta o trabalho educacional de planejamento e implementação dos cursos citados no começo deste texto.

\section{Referências}

ARNOLD, L., MEISELAS, L., ORGEL, G., PEMBERTON, L.B. Cross-disciplinary perspectives on a

liberal education for physicians. Pharos, v.47, n.1, p.24-8, 1984.

BARTOL, G.M. Using the Humanities in nursing education. Nurse Educ., v.11, n.1, p.21-3, 1986.

BECKINGHAM, C.R. Science, the humanities, nursing research and nursing. Int. Nurs. Rev., v.29, n.2, p.41-5, 1982.

BERGMAN, G.E., SORENSON, J.H., GABRIELSON, I.W. An adjunct Humanities faculty in Medical School. J. Med. Educ., v.57, n.5, p.413-15, 1982.

BERTMAN, S.L., MARKS, S.C. Humanities in medical education. Med. Educ., v.19, n.5, p.374-81, 1985.

BERTMAN, S. Ars Moriendi: illuminations on the good death from the arts and humanities. Hosp. J., v.13, n.1, p.5-28, 1998.

BIGGERSTAFF, D.E., SCHNITZ, G.W., WINGROVE, M.S. Enhanced communication skills for medical students through drawing. J. Biocommun., v.11, n.1, p.2-4, 1984.

BLIZEK, W.L., FINKLER, D. Teaching human values in Medicine. J. Med. Educ., v.52, n.10, p.85859, 1977.

BRESNAHAN, J.F., HUNTER, K.M. Ethics education at Northwestern University Medical School.

Acad. Med., v.64, n.12, p.740-43, 1989.

BRIEGER, G.H. Do the humanities make for humane physicians? (editorial) Pharos, v.57, n.3, p.42, 1994.

BRUDERLE, E.R. The arts and humanities: a creative approach to developing nurse leaders. Holist.

Nurs. Pract., v.9, n.1, p.68-74, 1994. 
BRUDERLE, E.R., VALIGA, T.M. Integrating the arts and humanities into nursing education. NLN Publ., v.14, Jun, p.117-44, 1994.

CRELLIN, J.K., BRIONES, A.F. Movies in Medical Education [letter]. Acad. Med., v.70, n.9, p.745, 1995.

DARBYSHIRE, P. Understanding the life of illness: learning through the art of Frida Kahlo.

Adv. Nurs. Sci., v.17, n.1, p.51-9, 1994.

DAVIS, S.K. Nursing and the humanities: health assessment in the art gallery. J. Nurs. Educ., v.31, n.2, p.93-4, 1992.

DICHI, I. O médico no cinema: uma nova abordagem na discussão de ética e psicologia médica.

Rev. Bras. Educ. Méd., v.18, n.1, p.40-1, 1994.

DOBBS, S.M. Learning in and through Art: a guide to discipline-based Art Education. Los Angeles: The Getty Education Institute for the Arts, 1998.

DOWNIE, R.S. Humane Medicine and literature. N. Z. Med. J., v.111, n.1058, p.1-2, 1998.

EVANS, M. Pictures of the patient: Medicine, science and humanities. Occas. Pap. R. Coll. Gen. Pract., v.76, Apr., p.1-16, 1998.

GOGEL, E.L., TERRY, J.S. Medicine as interpretation: the uses of literary metaphors and methods.

J. Med. Philos., v.12, n.3, p.205-17, 1987.

HARDISON, O.B. Problems of value in Medicine and the Humanities, or will the real doctor please stand up? Perspect. Biol. Med., v.20, n.3, p.439-49, 1977.

HAWKINS, A.H. Literature, medical ethics and "epiphanic knowledge". J. Clin. Ethics, v.5, n.4, p.283-90, 1994.

HOGG, P.L. Medical education now includes humanities. Pa Med., v.92, n.2, p.36-40, 1989.

HOOK, E.W. The Jeremiah Metzger Lecture. Humanities in medicine: treatment of a deficiency disorder. Trans. Am. Clin. Climatol. Assoc., v.108, p.203-21, 1996.

HOSHIKO, B. Nursing diagnosis at the art museum. Nurs. Outlook, v.33, n.1, p.32-6, 1985.

LAFFERTY, P.M. Balancing the curriculum: promoting aesthetic knowledge in nursing. Nurse Educ. Today, v.17, n.4, p.281-86, 1997

LODEN, K.C. Clinical experience at the museum of art. Nurse Educ., v.14, n.3, p.25-6, 1989.

MALACRIDA, R., WULLSCHLEGER, R., RAPIN, C.H. De la bioéthique aux "medical humanities" par l'éthique clinique. Rev. Med. Suisse Romande, v.118, n.12, p.1019-021, 1998.

MARSHALL, P.A., O'KEEFE, J.P. Medical students' first-person narratives of a patient's story of AIDS. Soc. Sci. Med., v.40, n.1, p.67-76, 1995.

MIDDLETON, J.A., SEN, P., MIDDLETON, J.R. Teaching humanistic behaviour: humanities study in the internal medicine residency. N. Jersey Med., v.90, n.10, p.763-66, 1993.

MOHR, W.K. Integrating esthetics into nursing: literature as a suggested modality. Arch. Psychiatr. Nurs., v.9, n.6, p.365-72, 1995.

MOORE, A.R. Medical Humanities - a new medical adventure. N. Engl. J. Med., v.295, n.26, p.1479-80, 1976.

MOORE, A.R. Medical humanities: an aid to ethical discussions. J. Med. Ethics, v.3, n.1, p.26-32, 1977.

MORANI, A.D. Art in medical education: especially plastic surgery. Esthet. Plast. Surg., v.16, n.3, p.213-218, 1992.

NOLL, J.G., LUFT, L., CHAVES, C.L., GUERCHFELD, M. Mesa-redonda: inter-relação da psiquiatria com letras e artes. Rev. Psiquiatr. Rio Grande do Sul, v.11, n.3, p.192-202, 1989.

PARKIN, C.J. Humanities for the health professional. AD Nurse, v.2, n.6, p.30-1, 1987.

PÁSZTOR, E. Parallels between three-dimensional thinking in neurosurgery and the development of perspective in art. Acta Neurochir. (Wien), v.124. n.2, p.176-78, 1993.

PECHANSKY, C., FURTADO, J., AMOM, S. Mesa Redonda: inter-relação da psiquiatria com 
letras e artes - 1. Rev. Psiquiatr. Rio Grande do Sul, v.11, n.3, p.185-91, 1989.

PEDEN, A.R., STATEN, R.R. The use of the humanities in psychiatric nursing education. J. Nurs.

Educ., v.33, n.1, p.41-2, 1994.

PELLEGRINO, E.D. The Humanities in medical education: entering the post-evangelical era. Theor.

Med., v.5, n.3, p.253-66, 1984.

PELOQUIN, S.M. Art: an occupation with promise for developing empathy. Am. J. Occup. Ther., v.50, n.8, p.655-61, 1996.

PHENIX, P.H. Realms of meaning. New York: McGraw-Hill Book, 1964.

PORTUGAL-ALVAREZ, J. La enseñanza del Humanismo Médico. Anal. Med. Interna, v.8, n.11, p.569-70, 1991.

SIEGEL, M., SIEGEL, C. The use of literature in professional nursing education. Nurs. Forum, v.16, n.2, p.156-64, 1977.

SMITH, R.A. The changing image of art education: theoretical antecedents of Discipline- Based Art Education. In: SMITH, R. A. (Ed.) Discipline-based Art Education: origins, meaning, and development. Chicago: University of Illinois Press, 1989. p.3-34.

SWEENEY, B. The place of the humanities in the education of a doctor. Br. J. Gen. Pract., v.48, n.427, p.998-1102, 1998.

TAYLOR, R.N. Medical humanities and clinical practice. J. South Carol. Med. Assoc., v.91, n.12, p.500-2, 1995.

VANDE-ZANDE, D.A. The liberal arts and professional nursing: making the connections.J. Nurs. Educ., v.34, n.2, p.93-4, 1995.

VOGEL, S. The importance of visual arts in the life of a neurosurgeon. Acta Neurochir. (Wien), v.124, n.2, p.168-71, 1993.

WILSON, H.S. A case for humanities in professional nursing education. Nurs. Forum, v.13, n.4, p.406-17, 1974.

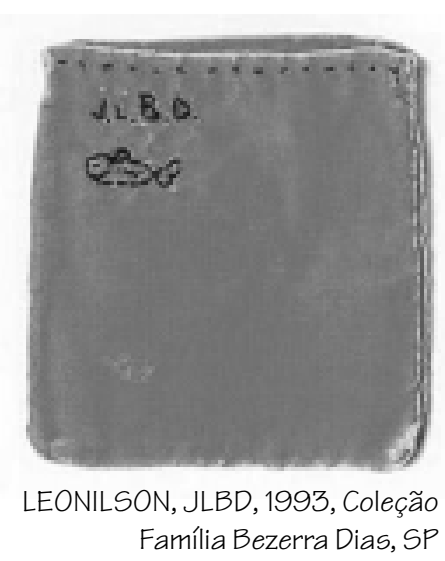

TAPAjÓS, R. La introducción del arte en los currículos médicos, Interface _ Comunic, Saúde, Educ, v.6, n.10, p.27-36, 2002.

La capacitación profesional apropiada en el campo de la infección por HIV/ Sida asume que el tratamiento y los cuidados sean ofrecidos de forma adecuada, ética y humanizada. La preocupación con esta capacitación ha acentuado la discusión sobre cómo la formación médica puede integrar la adquisición de la excelencia técnica y rasgos humanistas. Así, la educación medica pasa a incorporar el desafío de adecuarse para providenciar a los médicos, productos finales de sus currículos, una formación humanista y humanizadora. Las humanidades, especificamente las Artes, son disciplinas que clásicamente cumplen esta función. Su introducción en los currículos médicos se postula y se discute, sea por su valor intrínseco, sea para facilitar la ejecución de objetivos más amplios dentro de esos currículos.

PALABRAS-CLAVE: Educación médica; Humanismo, Artes; HIV/SIDA. 